\title{
Contents, Vol. 93, 1960
}

International Review of Gastroenterology - Revue internationale de Gastroenterologie Internationale Zeitschrift für Gastroenterologie

Als «Archív für Verdauungskrankheiten» gegründet von J. Boas (1895-1933)

Fortgeführt von P. Morawitz (1934-1936), N. Henning, A. Ltiger, R. Staehelin (1936-1939)

R. Staehelin und A. Hurst (1939-1942), A. Hurst und W. Löffler (1942-1944)

Offizielles Organ der Schweizerischen Gesellschaft für Gastroenterologie Organe Officiel de la Société suisse de Gastro-entérologie

Offizielles Organ der Deutschen Gesellschaft für Verdauungs-und Stoffwechsel-Krankheiten

H. Babtelheimer, Berlin

H. H. BERG, Hamburg

R. BOLLER, Wien

B. M. VON BONSDORFF, Helsinki

M. COPPO, Modena

Ch. Debray, Paris

Harold C. Edwards, London

A. FrOEHLICH, Anvers

F. Gallart-Monès †, Barcelona

A. J. HAEX, Leiden E. HAFTER, Zurich K. HERFOHT, Praha

EDITORES:

H. Kalk, Kassel

C. KATSCH, Greifswald

I. Kleeberg, Jerusalem

A. LAMBLING, Paris

C. D. DE LANGEN, Utrecht

M. LÜDIN, Basel

C. Marina-Fiol, Madrid N. G. Markoff, Ch ur

J. MASSION, Bruxelles

E. Meulengracht, København

H. G. MOGENA, Madrid

F. MOUTIER, Paris

R. NISSEN, Basel

L. Norpoth, Essen

H. M. POLLARD, Ann Arbor, Mich.

J. F. PONTES, São Paulo

H. POPPER, New York, N.Y.

R. PrÉVÔT, Hamburg

M. ROYER, Buenos Aires

G. SOTGIU, Bologna

N. SVARTZ, Stockholm 
W. VOLWILER, Seattle, Wash.

L. ZUKSCHWERDT, Hamburg

REDACTORES: N. HENNING - Th. C. HUNT - B. IHRE - W. LÖFFLER SECRETARII: L.

Demling - M. Demole - H. Kapp

Vol. 93

1960

BASEL (Schweiz) S. KARGER NEW YORK

INDEX

Aagesen, G.: Treatment of Pain Induced by Tumor Metastasis, According to Dogliotti.. 119

Avery Jones, F.: Some Observations on Idiopathic Procto-Colitis 107

Benkö, G.: Die gastroskopische Beurteilung des pathologischen Zustandes der Magen-

geschwüre 396

Boss, J. H.: vide Leffkowitz, M.

Brante, G.; Groth, O.; Sjöberg, S.-G. and Stenninger, G.: Iron Absorption and Storage

in Sideropenia 113

Camarri, E.; Verme, G. and Gioannini, P.: Liver Changes in Chronic Gastroduodenitis 385

Clémençon, G. H.; Ihre, B. J. E. and Plengier, L. H.: Hiatal Hernia in Bronchial Asthma 337

Coninx, G.: vide Heinkel, K.

Djaldetti, M.: vide Freud, M.

Elster, K.: vide Heinkel, K.

Ericson, S. M.: ACTH in the Treatment of Acute Postoperative Pancreatitis 129

Freud, M.; Djaldetti, M.; Vries, A. de and Leffkowitz, M.: Postcholecystectomy Syn

drome: A Survey of 114 Patients after Biliary Tract Surgery 288

Fric, P.; Herfort, K. and Zeman, J.: The Chromodiagnostic Biliary Intubation with the

Use of Methylene Blue 210

Friedländer, E.: vide Loewenthal, M.

Fucík, M.; Ronský, R. und Skála, I.: Die Bestimmung der Pepsinaktivität im Magen-

saft, Serum und Urin bei Patienten mit Geschwürskrankheit ... 79

Gioannini, P.: vide Camarri, E.

Groth, O.: Dissecting Aortic Aneurysm Simulating Acute Abdominal Cases

120

Groth, O.: vide Brante, G.

Heinkel, K.; Landgraf, J.; Elster, K.; Henning, N. und Coninx, G.: Häufigkeit und Bedeutung von Becherzellen in der menschlichen Magenschleimhaut. Ergebnisse klinischer und bioptischmorphologischer Untersuchungen 269

Heinkel, K.: vide Schmid, E.

Heiwinkel, H.; Lindvall, S. and Reizenstein, P.: Gastro-Intestinal Activity of Cellu-

lase in Man after Oral Administration

69

Henning, N.: H. H. Berg zum 70. Geburtstag

Henning, N.: vide Heinkel, K.

Henning, N.: vide Schmid, E.

Herfort, K.: vide Fric, P.

Hofstetter, J. et Mosimann, R.: Résultats immédiats et éloignés de la gastrectomie totale

pour cancer de Гestomac 193

Ihre, B. J. E.: vide Clémençon, G. H.

Jimenez-Diaz, C.; Linazasoro, J. M.; Marina, C. and Romeo, J. M.: Studies on Idio

pathic Steatorrhea with Fat Labeled (I131) 228 
Krentz, K.: Uber die Behandlung chronischer Lebererkrankungen mit Rohleberextrakt 300

Labò, G.: vide Sotgiu, G.

Landau, B.: vide Leffkowitz, M.

Landgraf, J.: vide Heinkel, $\mathrm{K}$.

Leffkowitz, M.; Landau, B. and Boss, J. H.: Insulinoma Associated with Peptic Ulcers.

Report of a Case 157

Leffkowitz, M.: vide Freud, M.

Linazasoro, J. M.: vide Jimenez-Diaz, C.

Lindvall, S.: vide Heiwinkel, $H$.

Loewenthal, M.; Steinitz, H. und Friedländer, E.: Gastritis hypertrophica gigantea und Magenkarzinom 133

Marina, C.: vide Jimenez-Diaz, C.

Monges, H.: Etude concernant les pressions intra-oesophagiennes et intra-gastriques chez des sujets normaux $\quad 46$

Mosimann, R.: vide Hofstetter, J.

Palmer, W. L.: Cancer of the Stomach. A Brief Review of the Problem 5

Piængiér, L. H.: vide Clémençon, G. H.

Polak, M. and Pontes, J. F.: Transhepatic Cholangiography with the Aid of Laparoscopy.

Preliminary Report on a New Technique

65

Pontes, J. F.: vide Polak, M.

Reizenstein, P.: vide Heiwinkel, $H$.

Rissier, H. L. jr. : Hemangiomatosis of the Intestine. Discussion Review of the Literature and Report of Two New Cases 357

Roisch, R.: vide Sprung, H. B. Romeo, J. M.: vide Jimenez-Diaz, C. Ronský, R.: vide Fuc¹/8, M.

Royer, M.: La mucosographie duodénale dans le diagnostic des duodénites

Schmid, E.; Seng, I.; Henning, N. und Heinkel, K.: 5-Hydroxytryptamin und Erkran-

kungen der Verdauungsorgane. Ergebnisse biologischer Blutanalysen

sowie klinischer und bioptisch-morphologischer Untersuchungen.... 235 Semin, R. N.:

Splenoportography and Intrasplenic Manometry in Hepatic Bilharziasis . .86,167 Seng, I.: vide

Schmid, E.

Sjöberg, S.-G.: Tourist's Disease 120

Sjöberg, S.-G.: vide Brante, G.

Skála, I.: vide Fucík, M.

Sotgiu, G.; Labô, G. and Vannini, P.: Study of Fat Fractions of the Stools in Steatorrheas.

Clinical and Experimental Studies 27

Sprung, H. B. und Roisch, R.: Über die Registrierung der Dünndarmmotilität mit dem

Intestinalsender $\quad 145$

Steinitz, H.: vide Loewenthal, M.

Stenningeh, G.: vide Brante, G.

Vándorfy, J.: Klinische Beobachtungen über den Einfluß der Ovarialfunktion auf den

Verlauf der gastroduodenalen Geschwürskrankheit 294

Vannini, P.: vide Sotgiu, G. Verme, G.: vide Camarri, E. Vries, A. de: vide Freud, M.

Wahren, H.: Corticotropin in Acute Cholecystitis 118

Walters, W.: Six to Ten-Year Follow-Up of the Surgical Treatment of Duodenal, Gastric

and Gastrojejunal Ulcer

15

Zeman, J.: vide Fric, P. 
GESELLSCHAFTSBERICHTE - SOCIETY TRANSACTIONS - SOCIÉTÉS

Swedish Society of Gastroenterology, Eskilstuna, Oct. 18th, 1958107

EDITORIAL Papillomes et cancers cholédociens

180

Cuivre, foie et système nerveux 255

Conception actuelle du traitement de la côlite ulcéreuse

La soi-disant petite insuf fisance hépatique $\quad 405$

322

SEITE DER THERAPIE - PAGE DE THÉRAPEUTIQUE 56,121,181, 257, 324,408

REFERATE - ABSTRACTS - ANALYSES

I. Allgemeines - Généralités

57

II. Oesophagus

60

Hernia hiatalis

61

Magen - Estomac

62

Ulcus $\quad 62$

Carcinoma 63

Chirurgie 123

Varia 125

V. Leber - Foie

Diagnostik 182

182

Cirrhose $\quad 184$

Varia 188

VI. Pancreas 259, 325

VII. Gallenwege - Voies biliaires 409

ZEITSCHRIFTENUBERSICHT-JOURNAL REVIEW-REVUE DES REVUES 265, 334,414

KONGRESSKALENDER - COMING MEDICAL MEETINGS - TABLEAU DES CONGRÈS 192

NECROLOGIA $\quad 336$

BUCHBESPRECHUNGEN - BOOK REVIEWS - LIVRES NOUVEAUX 64,127,190, 264, 333

VARIA $\quad 128,192,268,336$

Alle Rechte, insbesondere das de $\Gamma$ Übersetzung in frem,de Sprachen, vorbehalten.

Ohne ausdrückliche Genehmigung des Verlages ist es auch nicht gestattet, diese Zeitschrift

oderTeile daraus

auf photomechanischem Wege (Photokopie, Mîkrokopie) zu vervielfältigen.

(C)

Copyright 1960 by S. Karger AG, Basel.

Printed in Switzerland by Bnchdruckerei Werner \& Bischoff AG, Basel.

Cliches: Aberegg-Steiner \& Cie., AG, Bern, und Steiner \& Cie., AG, Basel. 